\title{
El rol del supervisor de campo clínico en Enfermería: perspectiva estudiantil
}

\section{The Role of the Clinical Field Supervisor in Nursing: A Student Perspective}

\author{
O papel do supervisor de campo clínico na enfermagem: uma perspectiva estudantil
}

\author{
Yerty Otárola Millar ${ }^{1}$, ORCID 0000- 0002-9193-4030 \\ Mónica Illesca Pretty ${ }^{2}$, ORCID 0000-0003-0635-5331 \\ Alejandro Antonio Hernández Díaz ${ }^{3}$, ORCID 0000-0003-3380-2846 \\ ${ }^{1}$ Universidad Autónoma de Chile \\ ${ }^{2}$ Universidad de La Frontera, Chile \\ ${ }^{3}$ Escuela de Enfermería, Facultad de Ciencias, Universidad Mayor, Chile
}

Resumen: Objetivo: Conocer la opinión de estudiantes de la carrera de Enfermería de la Universidad Autónoma de Chile, respecto del rol del supervisor clínico. Metodología: Investigación cualitativa, descriptiva, interpretativa a través de un estudio intrínseco de caso. Muestra intencionada, de casos por criterios y por conveniencia, conformada por 24 estudiantes de quinto año, previa firma del consentimiento informado, año 2015. Recolección de datos mediante tres grupos focales; para el análisis de ellos se utilizó reducción progresiva y agrupación en categorías descriptivas. Se utilizaron los criterios de rigor de Guba, triangulando por investigadores. Aspectos éticos resguardados por el Comité Ético de Universidad de La Frontera. Resultados: En el Nivel 1 emergieron 257 unidades de significado, agrupadas en: "Factores obstaculizadores del supervisor clínico", "Características del supervisor clínico", "Factores facilitadores del supervisor clínico" y "significado del supervisor clínico". En el Nivel 2 surgen tres núcleos temáticos, finalizando en el Nivel 3 con dos dominios cualitativos: "Factores asociados al supervisor clínico que inciden en el proceso enseñanza-aprendizaje" y "Perfil y atributos del supervisor clínico". Conclusiones: Para los educandos es importante la formación docente y disciplinar de quien realiza la supervisión clínica, destacando las habilidades interpersonales para ejercer el rol.

Palabras claves: estudiantes de enfermería; práctica del docente de enfermería; enfermería.

Abstract: Objective: Determine the opinion of students at the School of Nursing of Universidad Autónoma de Chile, about the role of the Clinical Supervisor. Methodology: Qualitative, descriptive, exploratory, interpretive through an intrinsic case study research. Intentional sample, cases by criteria and by convenience, formed by 24 fifth year students, after signing the Informed Consent in 2015. Data collection was through three focus groups and analysis through progressive reduction and descriptive grouping in categories. Guba rigor criteria was used, triangulating by researchers. The ethical aspect was guard by the 
Universidad de la Frontera's Ethics Committee. Results: Level 1 analysis emerged 257 units of meaning, grouped into: "Hindering Clinical Supervisor Factors", "Clinical supervisor characteristics", "Facilitating Clinical Supervisor Factors" and "Meaning of the Clinical Supervisor". In Level 2 three thematic nuclei arise, ending with two Level 3 qualitative domains: "Factors associated with the Clinical Supervisor that affect the teaching-learning process" and "Profile and attributes of the Clinical Supervisor". Conclusions: For students, the teaching and disciplinary training of the person who performs clinical supervision is important, highlighting the interpersonal skills to exercise the role.

Keywords: nursing students; nursing faculty practice; nursing.

Resumo: Objetivo: Conhecer a opinião de estudantes da Carreira de Enfermagem da Universidad Autónoma de Chile sobre o papel do supervisor clínico. Metodologia: Pesquisa qualitativa, descritiva, interpretativa, através de um estudo de caso intrínseco. A amostra, intencional, de casos por critério e por conveniência, composta por 24 alunos do quinto ano, assinatura prévia do Termo de Consentimento Livre e Esclarecido, 2015. Coleta de dados: três grupos focais; para analisá-los, foram utilizados redução progressiva e agrupamento em categorias descritivas. Foram utilizados os critérios de rigor de Guba, triangulados pelos pesquisadores. Aspectos éticos protegidos pelo Comitê de Ética da Universidad de la Frontera. Resultados: No Nível 1, surgiram 257 unidades de significado, agrupadas em: "fatores obstrutivos do supervisor clínico", "características do supervisor clínico", "fatores facilitadores do supervisor clínico" e "significado do supervisor clínico". No nível 2 surgem três núcleos temáticos, terminando no nível 3 com dois domínios: fatores associados ao supervisor clínico que afetam o processo de ensino-aprendizagem e perfil e atributos do supervisor clínico. Conclusões: Para os alunos, é importante o treinamento docente e disciplinar daqueles que realizam a supervisão clínica, destacando as habilidades interpessoais para o exercício da função.

Palavras-chave: estudantes de enfermagem; prática do professor de enfermagem; enfermagem.

Recibido: 20/02/2020

Aceptado: 28/06/2021

Cómo citar:

Otárola Millar Y, Illesca Pretty M, Hernández Díaz AA. El rol del supervisor de campo clínico en Enfermería: perspectiva estudiantil. Enfermería: Cuidados Humanizados. 2021;10(2):42-57. DOI: 10.22235/ech.v10i2.2082

Correspondencia: Alejandro Antonio Hernández Díaz. E-mail: alejantohd@ hotmail.com

\section{Introducción}

En la formación de los enfermeros las actividades prácticas de la gestión del cuidado ocupan un porcentaje importante en el Plan Curricular. ${ }^{(1)}$ Estas experiencias clínicas se constituyen en uno de los principales quehaceres para la adquisición de las competencias 
disciplinares y genéricas. ${ }^{(2,3)}$ En este aspecto cobra relevancia el docente que hace la labor de supervisor, ya que mediante el proceso enseñanza-aprendizaje debe proporcionar herramientas y elementos hegemónicos en la preparación profesional ${ }^{(4,5)}$ de tal forma de cumplir con el perfil de egreso, logrando brindar asistencia integral al ser humano en los diferentes niveles de atención. ${ }^{(6)}$

Su rol es de vital importancia, ya que se configura como arquetipo académico para resguardar no solo la integración del aspecto teórico con la atención directa al usuario, sino también velar por la creación de un ambiente favorable y la correspondiente evaluación de las actividades propuestas. ${ }^{(7)}$ En otras palabras, son las personas implicadas en el acompañamiento, consejo, orientación y evaluación de los estudiantes durante sus prácticas clínicas, a través de una relación centrada en las dimensiones de exigencia, trabajo y emociones. ${ }^{(8)}$ Por otro lado, desde lo clínico muchas veces al docente le corresponde: seleccionar los pacientes según los objetivos del programa, prestar el cuidado con los estudiantes, promover la reflexión de la experiencia y actuar, sobre todo, como elemento facilitador en el proceso de aprendizaje. ${ }^{(9)}$

En este aspecto es importante destacar que el ambiente de aprendizaje clínico es complejo y multifuncional, ello debido a que se produce una interacción focalizada en atención directa entre usuarios, equipo de salud, familiares y ambiente, ${ }^{(10)}$ lo que genera estrés tanto en los estudiantes como en el supervisor clínico. ${ }^{(11)}$

El supervisor clínico se define como un profesional de enfermería que asume de forma voluntaria la responsabilidad del aprendizaje práctico clínico, en escenarios reales de los estudiantes a su cargo. Este es su referente y soporte pedagógico de una manera planificada y coordinada en la aproximación al papel profesional y a su capacidad para constituirse como profesionales autónomos, con capacidad resolutiva y con una actitud positiva hacia el ejercicio de su profesión. ${ }^{(12)}$ Además, debe demostrar capacidades en los dominios del saber aprender (autorregulación de la propia práctica y al trabajo conjunto con otros académicos); del saber (investigación para la producción de conocimiento, teorías y metodologías educativas, así como la cultura organizacional de la institución); del saber hacer (destrezas didácticas para facilitar al educando el desarrollo de habilidades para otorgar cuidados), y del saber ser (óptimas relaciones interpersonales con sus pares y discípulos, de modo de constituirse como modelo de formación). ${ }^{(13,14)}$

Algunos estudios concluyen que se debe fortalecer la función del supervisor clínico por medio de la educación continua, dirigida al uso de habilidades y experiencias apartándose del modelo puramente repetitivo y carente de reflexión. Debe generar canales de comunicación verbal y no verbal, fomentar el estudio en grupos colaborativos, asumir la responsabilidad de implementar estrategias de aprendizaje de acuerdo con las necesidades de cada estudiante, guiar en el proceso de aprender en las prácticas clínicas y evaluar en forma permanente el logro de los objetivos planteados. $(2,15,16)$

También es importante señalar que se ha demostrado que la integración docente/asistencial es un elemento constructivo y facilitador del proceso de enseñanzaaprendizaje $^{(9,17)}$ y que las competencias genéricas, son el rasgo fundamental de quien realiza la labor de supervisor clínico. ${ }^{(18,19)}$

Las universidades tienen la labor de mantener una actitud de cambio y transformación permanente, con altos valores solidarios, éticos, estéticos, humanitarios y de amor por la profesión. Estos, unidos a la docencia, asistencia e investigación, hacen del estudiante un profesional integral, capaz de aplicar el Proceso de Atención de Enfermería 
como método científico para dar solución a los problemas y necesidades que afectan la salud de la población, ello plasmado en el ambiente clínico como eje de la supervisión. ${ }^{20,21,22)}$

Por esto, la formación universitaria como educación formal debe preocuparse de la persona en su integridad, como ser en desarrollo y como sujeto social. Posee competencias profesionales quien dispone de los conocimientos, las destrezas y las actitudes necesarias para ejercer una profesión, puede revisar los problemas profesionales de forma autónoma y flexible y está capacitado para colaborar en su entorno profesional y en la organización del trabajo. ${ }^{(23,24)}$ La competencia es una disposición para enfrentar eficazmente una familia de situaciones análogas, movilizando a conciencia y de manera rápida, pertinente y creativa múltiples recursos cognitivos: saberes, capacidades, microcompetencias, informaciones, valores, actitudes, esquemas de percepción, evaluación y razonamiento. ${ }^{(25,26)}$

Por lo anterior, es deber institucional que se abran espacios reflexivos en la valoración permanente de las experiencias estudiantiles en voz de estos, ya que ello propicia mecanismos evaluativos para generar propuestas de mejora y, por esta razón, cobra relevancia estudiar esta perspectiva. Se plantea entonces como objetivo: conocer la opinión de estudiantes de la carrera de Enfermería de la Universidad Autónoma de Chile respecto al rol del supervisor clínico.

\section{Metodología}

La presente es una investigación bajo el paradigma cualitativo, exploratorio y descriptivo a través de un estudio intrínseco de casos. Las investigaciones cualitativas están interesadas en comprender la conducta humana desde el propio marco de referencia de quien actúa, permitiendo comprender en profundidad el problema y cuáles podrían ser sus causas, desde la mirada de los actores sociales. ${ }^{(27,28)}$ Los estudios exploratorios dan respuesta a la descripción del fenómeno, ${ }^{(29)}$ mientras que los de tipo descriptivo tienen significancia ya que no hay interpretación por parte del investigador sino una elaboración de las evidencias fidedignas de los participantes a la luz investigativa debidamente documentada. ${ }^{(30)}$

El estudio intrínseco de casos como diseño significa que no les interesa aprender sobre otros casos o sobre algún problema general, sino más bien necesita aprender sobre un caso particular. En este trabajo ha permitido profundizar la comprensión y especificidades del objeto de estudio, específicamente el caso es la carrera de Enfermería de la Universidad Autónoma de Chile. ${ }^{(30)}$

La muestra, intencionada de casos por criterios y por conveniencia, ${ }^{(32)}$ se conformó con 24 informantes claves, 6 hombres y 18 mujeres, con un promedio de edad de 25 años. $\mathrm{Su}$ criterio de inclusión fue: pertenecer al quinto año de la carrera de Enfermería de la Universidad, estar realizando la Práctica Profesional en el año 2015, tener disponibilidad horaria para asistir a las sesiones de grupos focales y haber firmado el Formulario de Consentimiento Informado.

Para la recolección de datos se utilizó la técnica cualitativa de grupo focal. ${ }^{(33)} \mathrm{Se}$ conformaron tres grupos de ocho integrantes cada uno, con una pauta guía para evitar que los temas claves no fueran explorados. Se consideró la participación de tres personas: una para registrar las notas de campo, otra para grabar la información y un investigador quien guio la técnica. Este daba el inicio con el enunciando de la pregunta orientadora, a través de la cual se intentó dar respuesta a la interrogante del estudio y enfocar la utilización de esta técnica de recogida de datos al logro de los objetivos planteados. Las sesiones se realizaron en dependencias universitarias y tuvieron una duración aproximada de 90 minutos. Para 
evitar los sesgos en las respuestas se utilizaron preguntas abiertas, para no emitir juicios, hablando claramente y de manera casual, evitando toda sugerencia que una respuesta pudiera ser más conveniente que otra, todo ello con la finalidad de no inducir a respuestas deseadas.

Con el objetivo de recoger las palabras exactas de los informantes y narrar en forma clara y completa lo que sucedió en el escenario, se utilizaron notas de campo y grabaciones de audio. Ambas técnicas aportaron datos específicos del tema abordado por el estudio, desde diferentes aproximaciones/contextos y apoyaron el logro de los objetivos.

Los datos se recopilaron hasta llegar al punto de saturación. Se adoptó el esquema de la reducción progresiva (separación de unidades, agrupamiento, identificación y clasificación de elementos), disposición, transformación y obtención de conclusiones verificables. ${ }^{(34)}$ El nivel progresivo de reducción y estructuración teórica fue a través de tres niveles:

- Nivel 1: Identificación de unidades de significado (narraciones textuales de los sujetos participantes) y su segmentación para la agrupación en categorías descriptivas.

- Nivel 2: A partir de las categorías descriptivas, construcción de un sistema de núcleos temáticos emergentes o metacategorías.

- Nivel 3: Identificación de los dominios cualitativos a través de un análisis secuencial y transversal de las metacategorías.

Las tres fases no fueron momentos diferenciados del proceso analítico, sino más bien diferentes operaciones (reducción de datos, disposición de datos y obtención de conclusiones) sobre el cuerpo de datos que configuraron un solo proceso infragmentable, recurrente, inductivo-deductivo y circular. En otras palabras, el proceso de análisis fue concurrente con la recogida de datos, sistemático, ordenado y flexible, lo cual refleja de algún modo el carácter holístico de la investigación cualitativa. (34)

La rigurosidad científica estuvo determinada por cuatro criterios: valor de verdad o credibilidad (triangulación por investigador, comprobación con participantes del estudio), aplicabilidad o transferibilidad (recogida abundante de información y descripción minuciosa), consistencia o dependencia (réplica paso a paso) y neutralidad o confirmabilidad (consenso con otros investigadores, juicio crítico de experto). ${ }^{(26,35,36)}$ Se contó con el aval del Comité de Ética de una entidad universitaria externa. Se efectuó además triangulación por investigador y comprobación con los participantes. ${ }^{(36)}$

Respecto al análisis del rigor ético, se consideran los postulados de Ezequiel Emannuel, ${ }^{(37)}$ se matiza el requisito respecto a los sujetos inscritos al incluir aspectos relacionados con balance riesgo-beneficio y protección de la confidencialidad, mediante la presentación y aprobación del Comité Científico de Ética de la Universidad de la Frontera.

\section{Resultados}

El análisis de los datos derivados de los grupos focales siguió un esquema que en forma progresiva generó una reducción de la información concordante con las interrogantes del estudio, a través de tres niveles: 
Nivel 1. Se realizó segmentación e identificación de unidades de significado de las narraciones textuales proporcionadas por los informantes claves y agrupación en categorías descriptivas de los fragmentos de texto (codificación) con sentido semántico relacionado directamente con el carácter reflexivo desde el punto de vista del informante.

Para ello, se inició una primera lectura de todas las transcripciones, lo que permitió tener una idea global del contenido de estas y conocer los temas nucleares en torno a los cuales se articulaba el discurso de los participantes.

En una segunda lectura se segmentaron los datos, para lo cual se utilizó el criterio de tema abordado, por tanto, se extrajeron y emergieron las distintas categorías donde se incluyen las unidades de significado. Con ello se seleccionaron las que eran relevantes para los objetivos del estudio descartando aquellas que no tenían ningún tipo de relación.

En resumen, los procesos metodológicos de categorización y segmentación fueron dos operaciones que se realizaron simultáneamente, de esta forma las categorías se identificaron con códigos. El proceso de construcción de categorías fue inductivo, abierto y generativo, utilizando como guía la pauta de entrevista y los objetivos de la investigación.

Al final de este proceso se encontraron 257 unidades de significado relevantes para el estudio agrupado en 4 categorías emergentes que se definen en la Tabla 1. En las tablas subsiguientes se presentan los resultados en relación con cada una de las categorías identificadas.

\section{Tabla 1. Categorías emergentes del estudio}

\begin{tabular}{|c|c|c|c|c|}
\hline \multirow[t]{2}{*}{ N. ${ }^{\circ}$} & \multirow[t]{2}{*}{ Código } & Categorías emergentes codificadas & \multicolumn{2}{|c|}{$\begin{array}{c}\text { Frecuencia unidades } \\
\text { de significado }\end{array}$} \\
\hline & & Factores obstaculizadores del supervisor clínico & $\begin{array}{c}\mathrm{n} \\
89\end{array}$ & $\begin{array}{c}\% \\
34.6\end{array}$ \\
\hline 2 & CSCL & Características del supervisor clínico & 84 & 32.7 \\
\hline 3 & FFSC & Factores facilitadores del supervisor clínico & 45 & 17.5 \\
\hline 4 & SSC & Significado del supervisor clínico & 39 & 15.2 \\
\hline \multicolumn{2}{|c|}{ TOTAL } & & 257 & 100 \\
\hline
\end{tabular}

Fuente: Elaboración propia (2020) 
Tabla 2. Distribución de frecuencias unidades de significado de la categoría Factores obstaculizadores del supervisor clínico (FOSC)

\begin{tabular}{|c|c|c|c|}
\hline \multirow[t]{2}{*}{ Código } & \multirow[t]{2}{*}{$\begin{array}{l}\text { Categoría } \\
\text { FOSC }\end{array}$} & \multicolumn{2}{|c|}{$\begin{array}{l}\text { Frecuencia unidades } \\
\text { de significado }\end{array}$} \\
\hline & & $\mathrm{n}^{\circ}$ & $\%$ \\
\hline \multirow{6}{*}{$\begin{array}{l}\text { Relaciones } \\
\text { interpersonales } \\
\text { RINT }\end{array}$} & Mal trato & 16 & 18,0 \\
\hline & Desinterés & 9 & 10,1 \\
\hline & $\begin{array}{l}\text { Comunicación verbal/gestual } \\
\text { inadecuada }\end{array}$ & 6 & 6,7 \\
\hline & Sin autocrítica & 4 & 4,5 \\
\hline & Intolerante & 3 & 3,4 \\
\hline & $\begin{array}{l}\text { Sin experiencia y conocimiento área } \\
\text { que supervisan }\end{array}$ & 8 & 9,0 \\
\hline \multirow{5}{*}{$\begin{array}{l}\text { Formación docente } \\
\text { FDO }\end{array}$} & No dan retroalimentación & 7 & 7,9 \\
\hline & Diferentes criterios de evaluación & 7 & 7,9 \\
\hline & $\begin{array}{l}\text { Desconocimiento teórico de } \\
\text { docencia }\end{array}$ & 6 & 6,7 \\
\hline & Incumplimiento de objetivos & 4 & 4,5 \\
\hline & Económico & 3 & 3,4 \\
\hline Factores personales & Saliente de turno & 3 & 3,4 \\
\hline \multirow[t]{2}{*}{ FPER } & Edad & 3 & 3,4 \\
\hline & Uso celular & 1 & 1,1 \\
\hline \multirow{3}{*}{$\begin{array}{l}\text { Factor institucional } \\
\text { FINS }\end{array}$} & Excesiva rotación docente & 6 & 6,7 \\
\hline & Cantidad de alumnos por docente & 2 & 2,2 \\
\hline & Docentes de otro establecimiento & 1 & 1,1 \\
\hline TOTAL & & 89 & 100 \\
\hline
\end{tabular}

Fuente: Elaboración propia (2020)

De los factores obstaculizadores, las relaciones interpersonales fueron las más mencionadas en los discursos. Destacan los rangos medios de formación docente y factores personales, en tanto el factor institucional fue la menos mencionada. 
Tabla 3. Distribución de frecuencias unidades de significado de la categoría Características del supervisor clínico (CSCL)

\begin{tabular}{llcc}
\hline Código & Categoría & \multicolumn{2}{c}{$\begin{array}{c}\text { Frecuencia unidades } \\
\text { de significado }\end{array}$} \\
\hline & CSCL & $\mathrm{n}^{\mathbf{o}}$ & $\%$ \\
& Buen trato & 10 & 12,0 \\
Competencias Genéricas & Empatía & 10 & 12,0 \\
CGE & Confianza & 6 & 7,1 \\
& Autocrítico & 4 & 4,8 \\
& Responsabilidad & 4 & 4,8 \\
Características Personales & Exigente & 7 & 8,3 \\
CPER & Compromiso & 7 & 8,3 \\
& Motivador & 6 & 7,1 \\
Competencia Disciplinar & Presencia & 5 & 6,0 \\
CDIS & Experiencia clínica área a supervisar & 2 & 2,4 \\
Proceso Enseñanza- & Conocimiento & 6 & 9,4 \\
aprendizaje & Formación docente & 7,1 \\
PEAP & Retroalimentación & 7 & 7.1 \\
TOTAL & & 2 & 2.4 \\
\hline
\end{tabular}

Fuente: Elaboración propia (2020)

De las características del supervisor clínico destacan las competencias genéricas como las más visualizadas; de ellas se menciona en mayor medida el buen trato y empatía y de menor rango las relacionadas con el proceso enseñanza-aprendizaje orientado sobre todo en función de la retroalimentación ejercida por el supervisor clínico.

Tabla 4. Distribución de frecuencias unidades de significado de la categoría Factores facilitadores del supervisor clínico (FFSC)

\begin{tabular}{llcc}
\hline Código & Categoría & \multicolumn{2}{c}{$\begin{array}{c}\text { Frecuencia unidades } \\
\text { de significado }\end{array}$} \\
\hline & FFSC & $\mathrm{n}^{\mathbf{o}}$ & $\%$ \\
Actitud docente & Motivación & 12 & 26,7 \\
ADOC & Disposición & 7 & 15,6 \\
Relaciones interpersonales & Respeto & 1 & 2,2 \\
RINT & Empatía & 6 & 13,3 \\
Competencia disciplinar & Experiencia y conocimiento & 5 & 11,1 \\
CDIS & del área que supervisan & & 20,0 \\
Factor institucional FINS & Nivel de conocimiento & 2 & 4,4 \\
TOTAL & Contratado & 3 & 6,7 \\
\hline Futica & & 45 & 100 \\
\hline
\end{tabular}

Fuente: Elaboración propia (2020) 
Como factor facilitador resalta la actitud docente, y en específico lo concerniente a la motivación y disposición docente. Respecto al factor institucional, que es el de menos valoración en estas categorías, se visualiza la característica de contrato.

Tabla 5. Distribución de frecuencias de las unidades de significado de la categoría Significado del supervisor clínico (SSC)

\begin{tabular}{llcc}
\hline Código & Categoría & \multicolumn{2}{c}{$\begin{array}{c}\text { Frecuencia unidades } \\
\text { de significado }\end{array}$} \\
\hline & SSC & $\mathrm{n}^{\mathbf{~}^{\mathbf{0}}}$ & $\%$ \\
& Enseña (competencias) & 9 & 23,1 \\
Proceso de formación & Modelo & 4 & 10,3 \\
PFOR & Experiencia & 3 & 7,7 \\
& Retroalimenta & 2 & 5,1 \\
& Supervisa & 2 & 5,1 \\
Actitudes del supervisor & Guía & 8 & 20,5 \\
ACSU & Acompaña & 6 & 15,4 \\
TOTAL & Ayuda & 5 & 12,8 \\
\hline
\end{tabular}

Fuente: Elaboración propia (2020)

Con relación al significado del supervisor clínico se acentúa el proceso de formación en relación con las competencias en el acto de enseñar, la figura de modelo y, en menor grado, las actitudes propias del supervisor clínico, en específico respecto a la función guía y acompañamiento.

Nivel 2. Este nivel representa los núcleos temáticos emergentes o metacategorías que surgen del Nivel 1, agrupadas y estructuradas en las 4 categorías descriptivas codificadas que emergieron en ese nivel. Para ello se realizó un proceso de comparación intercategorías en el que se buscaron similitudes estructurales y elementos comunes de este análisis. De ello emergieron 3 núcleos temáticos o metacategorías que representan la realidad estudiada tal como lo describen los informantes claves.

1. Factores que facilitan y obstaculizan el rol del supervisor clínico: Apreciación de los estudiantes con relación a aspectos que contribuyen y dificultan el rol del supervisor clínico, siendo las relaciones interpersonales y los factores institucionales coincidentes en ambos aspectos. Se destaca como facilitador la formación docente y factores personales, en tanto como obstaculizador la actitud del docente y la competencia disciplinar.

2. Competencias del supervisor clínico asociadas a la práctica del cuidado: Opiniones del estudiantado vinculadas a las competencias genéricas y disciplinares, como también a las características personales y a la formación docente para llevar a cabo el proceso enseñanza-aprendizaje.

3. Concepción de supervisor clínico: Valoración de los educandos con respecto al significado que tiene para ellos el supervisor clínico en su proceso de formación ya que les enseña, es un modelo, tiene experiencia, da retroalimentación y supervisa. También destacan en esta concepción la actitud que tiene ya que les guía, acompaña y ayuda. 
Nivel 3. Posterior al análisis secuencial y transversal de las metacategorías, se realizó una reconstrucción sintética desde una perspectiva ética, donde emergieron los dominios cualitativos. En cierto sentido todas las opiniones que expresaron los estudiantes participantes en este estudio están reflejadas en los dominios emergentes que se han definido. Sin lugar a duda que la negociación de significados e interpretaciones que se llevó a cabo en forma conjunta con los investigadores han contribuido en buena medida a este fin. Derivado de lo anterior, surgen dos dominios cualitativos:

\section{Factores asociados al supervisor clínico que inciden en el proceso enseñanza-} aprendizaje: Corresponde a la metacategoría 1 y se relacionan directamente con aquellos elementos que los estudiantes perciben como importantes en los supervisores clínicos, que van en directa relación con el proceso enseñanza-aprendizaje en los centros de práctica.

2. Perfil y atributos del supervisor clínico: Corresponde a las metacategorías 2 y 3 , y se relaciona directamente a los aportes que hacen los estudiantes con respecto a la definición del supervisor clínico y a las competencias genéricas y disciplinares que debieran tener para facilitar el proceso enseñanza-aprendizaje en la práctica del cuidado.

\section{Discusión}

Para los estudiantes, el significado del supervisor clínico se condice con las definiciones develadas en otros estudios: como la persona con mayor destreza en alguna área que pueda brindar apoyo y orientación. ${ }^{(2,7,8)}$

Otros autores indican que esta figura es la encargada de guiar, orientar, asesorar, enseñar y evaluar el aprendizaje de los estudiantes. ${ }^{(9-11)}$ Dichas apreciaciones se pueden atribuir a que, al ser una experiencia de aprendizaje estresante y que les provoca angustia y temor, necesiten mayor apoyo para enfrentarla, requiriendo muchas veces de una persona que les demuestre capacidades en los dominios del saber aprender; del saber; del saber hacer, de modo de constituirse además como un modelo de formación, similar a lo encontrado en otros estudios y marco teórico. ${ }^{(10,12-16)}$

En relación a las características que debe tener un supervisor clínico, lo encontrado es coincidente con los discursos de otros estudios. ${ }^{(9,12,19,25)}$ En todos ellos se observa que deben tener competencias tanto genéricas como disciplinares y características personales como ser vocación, exigencia, compromiso, motivación y estar presente durante la supervisión. ${ }^{(12-14)}$ Otra particularidad develada por los estudiantes se relaciona con la formación docente que debe tener en el ámbito del proceso enseñanza-aprendizaje, a diferencia de otros autores que afirman que la relevancia en este aspecto son los atributos del modo de ser, comunicación, afecto, lenguaje, tiempo y espacio, entre otros elementos que se dan en esta fase académica. ${ }^{(15,16)}$

No fueron mencionados en este estudio factores potentes relacionados con la función de apoyo del docente clínico, quien es la figura que potencia el desarrollo de habilidades para el desempeño del trabajo emocional en los discentes en formación clínica. ${ }^{(40)}$

A su vez, lo encontrado en relación con las competencias genéricas y disciplinar (empatía, conocimiento) y proceso enseñanza-aprendizaje (formación docente, retroalimentación) es coincidente con lo develado en otros estudios. ${ }^{(10,14)}$ Ello refleja la importancia que los educandos le atribuyen a las personas implicadas en sus prácticas del 
cuidado para un aprendizaje significativo, resaltando que el rol no es solo la transmisión de conocimientos sino de guía, acompañamiento y de consejo en el proceso de aprender, por lo que amerita que este tenga formación continua en el área de la docencia. ${ }^{19,25)}$

Con respecto a las características personales, es coincidente con lo encontrado en otras investigaciones ${ }^{(14,16)}$ y amplía más la propuesta investigativa, ya que en ello se concibe que las características personales del rol del supervisor clínico permitan apoyar y complementar el proceso enseñanza-aprendizaje. ${ }^{(17)}$

No llama la atención que los informantes claves mencionan más factores obstaculizadores que facilitadores para quienes desempeñan la supervisión clínica. Lo encontrado se puede atribuir al factor estrés como un elemento que interfiere de forma importante en el proceso que viven los alumnos en sus prácticas clínicas. ${ }^{(7)}$ También a la angustia frente al desconocimiento de una determinada situación clínica, a la impotencia e incertidumbre ante estas y el riesgo a causar un daño sobre el paciente. ${ }^{(2,26)}$ En este punto, es importante mencionar que en el ambiente clínico los elementos negativos pueden deberse a que el educando espera del supervisor clínico un compromiso en diferentes aspectos no solo figurado como docente y competencias en ese ámbito, sino que además con un sentido de compañía, modelaje, guiamiento, liderazgo. ${ }^{(7,14)}$

Al igual que en otro estudio, las apreciaciones con relación a que el ambiente laboral es un factor negativo se pueden atribuir a que, en este nivel del Plan de Estudio, en base a sus experiencias son capaces de valorar estos ambientes, por lo tanto, frente a la evaluación constante y pertinente son capaces de dar respuesta a ello. ${ }^{(9)}$

No se identificaron factores obstaculizadores como déficit de formación docente, ${ }^{(38,39)}$ organización del tiempo dedicado a esta actividad en este estudio, ${ }^{(37)}$ ello fue mencionado en otras investigaciones relacionadas.

De los factores facilitadores destacan las actitudes docentes; los estudiantes le dan importancia a la motivación, lo que es coincidente con uno de los atributos que debe tener el supervisor clínico, ya que este debe motivarlos para asumir el profesionalismo que requiere demostrar frente al usuario, paciente y familia, como también debe estimular y tener una actitud de entusiasmo. ${ }^{(7,10)}$

$\mathrm{Al}$ igual que en otro estudio, la empatía y el respeto emergen mayoritariamente en las relaciones interpersonales, ${ }^{(14)}$ lo que refleja la correspondencia en los discursos de los informantes claves, ya que fue identificado como una de las características del supervisor clínico.

En la competencia disciplinar se observa congruencia en los discursos de los entrevistados, ya que la experiencia y conocimiento del área que supervisan han sido mayoritariamente mencionadas al igual que lo encontrado en otros estudios. ${ }^{(9,26)}$

De igual forma, es dable mencionar que el saber disciplinar permite que un individuo asimile el conocimiento y establezca relaciones causales entre diferentes sucesos, ${ }^{(25)}$ lo que conlleva al saber ejercido que consiste en la adquisición de una habilidad. ${ }^{(15,16)}$ Es decir, un individuo puede saber cómo se hace algo antes de descubrir su explicación y que a medida que el supervisor clínico adquiere experiencia, el conocimiento clínico se convierte en una mezcla de competencia práctica y teórica. ${ }^{(25,26)}$

\section{Conclusiones}

El estudiantado visualiza el rol del supervisor clínico como un eje de guía evidenciado en las prácticas. Lo reconocen como importante en el proceso de formación ya 
que promueve el desarrollo de competencias; son modelos a seguir, tienen actitudes de guía, experiencia, retroalimentan, supervisan, acompañan y ayudan en su aprendizaje clínico.

Las características que debe tener el supervisor clínico se enmarcan en: a) competencias genéricas: que les den un buen trato, que sean empáticos y que les den confianza; b) competencia disciplinar: experiencia clínica y conocimiento en el área a supervisar; c) características personales: vocación exigencia y compromiso y d) formación docente para ejecutar el rol.

Los aspectos facilitadores para la supervisión clínica son: actitud del docente, relaciones interpersonales, competencia disciplinar y algunos factores institucionales. En tanto los obstaculizadores: desempeño las relaciones interpersonales, formación docente, factores personales e institucionales.

Los resultados permiten la reflexión en forma sistemática y entender las prácticas educativas, de manera tal, que estas se traduzcan en una acción comprometida e informada desde el punto de vista teórico y práctico. estudiantes.

Limitaciones del estudio: acceso a la población y la disponibilidad horaria de los

\section{Referencias bibliográficas}

1. Moreno MA, Prado EN, García DJ. Percepción de los estudiantes de Enfermería sobre el ambiente de aprendizaje durante sus prácticas clínicas. Rev. Cuidarte [Internet]. 2013 [Consultado 04 mar 2019];4(1):444-449. Disponible en: http://www.scielo.org.co/scielo.php?script=sci_abstract\&pid=S221609732013000100003

2. Mansilla J, Ricouz A. Vivencia del rol docente clínico de enfermeras de hospitales del sur de Chile. Rev. Cienc. enferm. [Internet]. 2016 [Consultado 06 mar 2019];22(1):101111. Disponible en: https://scielo.conicyt.cl/scielo.php?script=sci_arttext\&pid=S071795532016000100009

3. Sánchez JR. Preparación pedagógica, docentes clínicos de enfermería y su relación con el proceso-enseñanza aprendizaje práctico. Rev cubana Enferm [Internet]. 2017 [Consultado 24 mar 2019];33(4). Disponible en: http://www.revenfermeria.sld.cu/index.php/enf/article/view/2131/297

4. Espinoza MB. Universidad Jaume I. Programa doctorado en Ciencias de la Enfermería. La evaluación de competencias clínicas en estudiantes de enfermería, un nuevo paradigma. Validación de rúbrica. [Tesis en Internet]. 2018 [Consultado 04 abr 2019]. Disponible https://www.tesisenred.net/bitstream/handle/10803/587111/2018_Tesis_Espinoza\%20 Fernandez_Maria\%20Bruna.pdf?sequence=1\&isAllowed $=y$

5. Sánchez Rodríguez JR. Preparación pedagógica, docentes clínicos de enfermería y su relación con el proceso-enseñanza aprendizaje práctico. Rev. Cubana Enferm [Internet]. 2017 [Consultado 22 may 2021];33(4). Disponible en: http://www.revenfermeria.sld.cu/index.php/enf/article/view/2131 
6. García M, Barbosa MA, Conz CA, Henrique M, Pinto MC, Muñoz LA. El cuidado en la Atención Primaria de Salud: vivencia de las enfermeras. Rev Reben. [Internet]. 2018 [Consultado 04 may 2019];71(supl1):574-581. Disponible en: http://www.scielo.br/pdf/reben/v71s1/es_0034-7167-reben-71-s1-0531.pdf

7. Vizcaya Moreno MF, Pérez Cañaveras RM, Jiménez Ruiz I, de Juan J. Percepción de los estudiantes de enfermería sobre la supervisión y entorno de aprendizaje clínico: un estudio de investigación fenomenológico. Rev. Enferm. glob. [Internet]. 2018 [Consultado 22 may 2021];17(51):306-331. Disponible en: https://scielo.isciii.es/scielo.php?script=sci_arttext\&pid=S1695-61412018000300011

8. Mastrapa YE, Gibert MP. Relación enfermera-paciente: una perspectiva desde las teorías de las relaciones interpersonales. Rev cubana Enferm [Internet]. 2016 [Consultado 26 may 2019];32(4). Disponible en: http://www.revenfermeria.sld.cu/index.php/enf/article/view/976/215

9. Betancourt 1, Muñoz LA, Merighi MA, Santos MF. El docente de enfermería en los campos de práctica clínica: un enfoque fenomenológico. Rev Latino-Amer Enferm [Internet]. 2011 [Consultado 04 jun 2019];19(5). Disponible en: http://www.scielo.br/pdf/rlae/v19n5/es_18.pdf

10. Carvajal Carrascal G, Laverde Contreras OL, Escobar Ramírez MC, Jaimes Carvajal N, Arroyo Marles LP. Ambiente de la práctica de enfermería y resultados del cuidado. Rev. Index Enferm [Internet]. 2016 [Consultado 04 jun 2019];25(1-2):14-17. Disponible en: http://scielo.isciii.es/scielo.php?script=sci_arttext\&pid=S1132$12962016000100004 \& \operatorname{lng}=\mathrm{es}$.

11. Morales E, Amigo C, Álvarez AJ. Estresores emocionales de estudiantes de enfermería en prácticas clínicas intrahospitalarias. Rev Empresa. [Internet]. 2018 [Consultado 26 jun 2019];(12)45:12-20. Disponible en: http://editorial.ucsg.edu.ec/ojsempresarial/index.php/empresarial-ucsg/article/view/126

DOI:10.23878/empr.v12i45.126

12. Carrasco Dois PA. Perfil de competencias del tutor clínico de enfermería desde la perspectiva del personal de enfermería experto. FEM (Ed. impresa) [Internet]. 2018 [Consultado 26 jun 2019];23(2):81-87. Disponible en: https://scielo.isciii.es/scielo.php?script=sci_arttext\&pid=S2014-98322020000200006

13. Millán Arteag EG. La práctica hospitalaria en los estudiantes universitarios de enfermería. Propuesta de protocolo de investigación. Rev. Dilemas contemp: educa, polít y valor [Internet]. 2021 [Consultado 22 may 2021];8(2):00012. Disponible en: http://www.scielo.org.mx/scielo.php?pid=S2007-

$78902021000100012 \&$ script=sci_arttext

14. Valenzuela S. La práctica de enfermería como foco de reflexión. Rev Aquichan. [Internet]. 2016 [Consultado 17 jul 2019];16(4):415-441. Disponible en: https://www.redalyc.org/pdf/741/74148832001.pdf 
15. Soto Núñez C, Avilés Reinoso L, Lucchini Rajes C, Soto fuentes P. Conociendo el rol del tutor clínico en profundidad. Rev. Ev. Investigación y Educación en Enfermería [Internet]. 2017 [Consultado 22 may 2021];35(3):356-363. Disponible en: http://www.scielo.org.co/scielo.php?pid=S0120-

$53072017000300356 \&$ script=sci_arttext\&tlng=es

16. Pulido M, Augusto JM, López E. Estudiantes de Enfermería en prácticas clínicas: El rol de la inteligencia emocional en los estresores ocupacionales y bienestar psicológico. Rev. Index de Enf. [Internet]. 2016 [Consultado 28 jul 2019];25(3):215-219. Disponible en: http://scielo.isciii.es/scielo.php?script=sci_arttext\&pid=S113212962016000200020

17. Sánchez S. Universidad Piloto de Colombia. Programa Especialista en Docencia Universitaria. El enfermero docente como evaluador en la práctica clínica de los estudiantes en el servicio de urgencias. [Tesis en Internet]. 2018 [Consultado 02 ago 2019]. Disponible

en: http://repository.unipiloto.edu.co/bitstream/handle/20.500.12277/4716/00005009.pdf? sequence $=1 \&$ is Allowed $=\mathrm{y}$

18. Caldas García DA. Capacidad didáctica del docente y logro de competencias durante sus prácticas clínicas en el interno de enfermería del hospital de emergencias Grau Essalud. Rev. Fac. Med. Hum. [Internet]. 2017 [Consultado 22 may 2021];21(2):378386. Disponible en: http://www.scielo.org.pe/scielo.php?script=sci_arttext\&pid=S230805312021000200378

19. Calderón MS. Competencias genéricas en enfermeras/os tituladas/os de la Universidad Arturo Prat, sede Victoria, 2010. Rev. Cienc y Enfer. [Internet]. 2012 [Consultado 23 ago 2019];18(1):89-97. Disponible en: https://scielo.conicyt.cl/scielo.php?script=sci_arttext\&pid=S071795532012000100009

20. Berrerril L. El humanismo en la formación y práctica de enfermería: una esperanza transformadora. Rev. Texto contexto - enferm. [Internet]. 2016 [Consultado 26 ago 2019];25(1). Disponible en: http://www.scielo.br/scielo.php?pid=S0104$07072016000100201 \&$ script $=$ sci_arttext\&tlng=es

21. Dehghani M, Ghanavati S, Soltani B, Aghakhani N, Haghpanah S. Impact of clinical supervision on field training of nursing students at Urmia University of Medical Sciences. Rev. J Adv Med Educ Prof. [Internet]. 2016 [Consultado 22 may 2021];4(2):88-92. Disponible

en: https://www.ncbi.nlm.nih.gov/pmc/articles/PMC4827761/

22. Ruiz E. La Enseñanza Clínica en Enfermería. Rev Mex Enf [Internet]. 2015 [Consultado 04 sep 2019];3:6-10. Disponible en: http://www.innsz.mx/imagenes/revistaenfermeria/septiembre-2015.pdf 
23. Aguilar Montoya C, Chaves Morales O, Loaiza Madrid C, Monge medina J, Vega Zúñiga MC, Rojas Arias JM, et al. Identificación del marco de competencias laborales en enfermería en el Sistema del Seguro Social Costarricense. Rev. Enferm Actual de Costa Rica [Internet]. 2019 [Consultado 22 may 2021];(37):245-262. Disponibe en: http://www.scielo.sa.cr/scielo.php?script=sci_arttext\&pid=S1409$45682019000200245 \& \operatorname{lng}=\mathrm{en}$.

24. Galdeano C, Valiente A. Competencias profesionales. Rev. Educ. quím [Internet]. 2010 [Consultado 22 sep 2019];21(1):28-32. Disponible en: http://www.scielo.org.mx/scielo.php?script=sci_arttext\&pid=S0187893X2010000100004

25. López E. En torno al concepto de competencia: un análisis de fuentes. Rev. de Curric y Form de Profeso. [Internet]. 2016 [Consultado 30 sep 2019];20(1):311-322. Disponible en: https://www.redalyc.org/pdf/567/56745576016.pdf

26. Fernández L, Díaz L, Leyva E. Competencias genéricas en docentes del nivel superior en el Estado de Guerrero. Rev. Iberoam para la Invest y el Des Educ. [Internet]. 2016 [Consultado 04 oct 2019];6(12). Disponible en: https://www.redalyc.org/pdf/4981/498153966020.pdf

27. Latorre A, Del Rincón D, Arnal J. Bases metodológicas de la investigación educativa. Barcelona: Hurtado; 1999.

28. Bedregal P, Besoain C, Reinoso A, Zubarew T. La investigación cualitativa: un aporte para mejorar los servicios de salud. Rev. méd. Chile [Internet]. 2017 [Consultado 07 sep 2019];145(3):373-379. Disponible en: https://scielo.conicyt.cl/scielo.php?script=sci_arttext\&pid=S003498872017000300012

29. Aguirre JC, Jaramillo LG. El papel de la descripción en la investigación cualitativa. Rev. Cinta de moebio, [Internet]. 2015 [Consultado 12 sep 2019];(53),175-189. Disponible en: $\quad$ https://scielo.conicyt.cl/scielo.php?script=sci_arttext\&pid=S0717554 X2015000200006

30. Arráez M, Calles J, Moreno L. La Hermenéutica: una actividad interpretativa. Rev. Sapiens. [Internet]. 2017 [Consultado 22 sep 2019];7(2):171-181. Disponible en: http://ve.scielo.org/scielo.php?script=sci_arttext\&pid=S1317-58152006000200012

31. Stake R. Investigación con estudio de casos. 4ta.ed. Madrid: Morata; 2007.

32. Otzen T, Manterola C. Técnicas de Muestreo sobre una Población a Estudio. Rev. Intern. Journal of Morpho. [Internet]. 2017 [Consultado 28 sep 2019];35(1):227-232. Disponible en: https://scielo.conicyt.cl/scielo.php?script=sci_arttext\&pid=S071795022017000100037

33. da Silveira Saldhana SDD, Colomé Beck CL, Weiller H, Nunes da Silva Fernandes M, Viero Viviani. Rev. Index Enfer [Internet]. 2015 [Consultado 22 may 2021];24(1-2):71- 
75. Disponible en: http://scielo.isciii.es/scielo.php?script=sci_arttext\&pid=S1132$12962015000100016 \& \operatorname{lng}=\mathrm{es}$.

34. Piza Burgos ND, Amaiquena Márquez FA, Beltran Baquerizo GE. Métodos y técnicas en la investigación cualitativa. Algunas precisiones necesarias. Rev. Conrado. [Internet]. 2019 [Consultado 22 may 2021];15(70):455-459. Disponible en: http://scielo.sld.cu/scielo.php?script=sci_arttext\&pid=S1990-

$86442019000500455 \& \operatorname{lng}=$ es\&tlng=es.

35. Guba E, Lincoln Y. Fourth generation evaluation. $2^{a}$ ed. London: Sage; 1990.

36. Noreña AL, Alcaraz N, Rojas JM, Rebolledo D. Aplicabilidad de los criterios de rigor y éticos en la investigación cualitativa. Rev. Aquichan. [Internet]. 2012 [Consultado 23 oct 2019];12(3):263-274. Disponible en: https://www.redalyc.org/pdf/741/74124948006.pdf

37. Acevedo Perez I, Rapiman ME, Cráneo orellana M, Rueda Castro L. Seven ethical requirements for quantitative and qualitative research nursing: experiences of three research ethics committees from Santiago, Chile. Rev. Inter de Human y Cien Soc. [Internet]. 2017 [Consultado 22 may 2021];7(7):19-24. Disponible en: http://repositorio.uchile.cl/handle/2250/147640

38. Hidalgo F, Latorre C, Leiva F. Universidad Austral de Chile. Facultad de Medicina. Escuela de Enfermería. Supervisión clínica a estudiantes de enfermería de la Universidad Austral de Chile: Experiencia de enfermeras/os del hospital base Valdivia. [tesis en Internet]. 2015 [Consultado 02 de ene 2020]. Disponible en: http://cybertesis.uach.cl/tesis/uach/2016/fmh632s/doc/fmh632s.pdf

39. Sánchez JR. P. Preparación pedagógica, docentes clínicos de enfermería y su relación con el proceso-enseñanza aprendizaje práctico. Rev cubana Enferm [Internet]. 2017 [Consultado 02 de ene 2020];33(4). Disponible en: http://www.revenfermeria.sld.cu/index.php/enf/article/view/2131/297

40. Diogo P, Rodrigues J, Sousa O, Martins H, fernandes N. Supervisão de estudantes em ensino clínico: Correlação entre desenvolvimento de competências emocionais e função de suporte. Revista Portuguesa de Enfermagem de Saúde Mental [Internet]. 2016 [Consultado 04 de ene 2020];(spe4):115-122. Disponible en: http://www.revenfermeria.sld.cu/index.php/enf/article/view/2131/297

Financiamiento: Artículo producto del Proyecto de Investigación Supervisores campo clínico: Opinión de estudiantes carrera Enfermería, Temuco-Chile 2015, estudio autofinanciado por autores. Tesis para optar al grado de Magíster en Enfermería con mención en Gestión del Cuidado, Universidad de La Frontera. Temuco, Chile, año 2015.

Contribución de los autores: a) Concepción y diseño del trabajo, b) Adquisición de datos, c) Análisis e interpretación de datos, d) Redacción del manuscrito, e) Revisión crítica del manuscrito. Y. O. M. ha contribuido en a, d, e; M. I. P. en b, c, d; A. A. H. D. en b, c, d.

\section{Editora científica responsable: Dra. Natalie Figueredo}

